\title{
ESTRATEGIAS DE ASOCIATIVIDAD PARA LA COMERCIALIZACIÓN DE PRODUCTOS AGROECOLÓGICOS
}

\section{STRATEGY FOR ASSOCIATIVITY OF AGRO-ECOLOGICAL PRODUCTS}

\author{
Lizzie Verónica Pazmiño Guevara ${ }^{1}$ \\ Jorge Rolando Álvarez Tello²
}

Recibido: 2021-06-05/ Revisado: 2021-07-01 / Aceptado: 2021-08-12 / Publicado: 2021-09-15

Forma sugerida de citar: Pazmiño-Guevara, L. V. y Álvarez-Tello, J. R. (2021). Estrategias de asociatividad para la comercialización de productos agroecológicos. Retos de la Ciencia. 5(e),112-123. https://doi.org/10.53877/rc.5.e.20210915.10

\section{RESUMEN}

La asociatividad tomó un papel protagónico, por los tiempos de crisis, de esta forma constituyó un aporte a los problemas de los pequeños productores del sector cooperativo y asociativo del modelo económico popular y solidario en el mercado actual. Este artículo está basado en el análisis de estrategias para potenciar la comercialización y marketing de alimentos agroecológicos que afrontó las nuevas exigencias entre productores y consumidores. Este trabajo fue desarrollado con carácter teórico, para el cual se realizó un amplio estudio bibliográfico de publicaciones, de los últimos cinco años, referentes a estrategias de asociatividad. Los resultados revelaron el sector popular y solidario investigado recurrían a la asociatividad como un modelo colectivo para incrementar su competitividad equitativa entre los grupos autónomos de pequeños y medianos productores. Asimismo; adoptaron estrategias de mejora continua para el trabajo cooperativo en red, la formación e integración social, la innovación tecnológica y el cambio de prácticas agrícolas. De ahí que, se concluye que la asociatividad constituyó una estrategia de fundamental importancia hacia el desarrollo cooperativo e integral de los grupos colectivos en el contexto investigado a desarrollar en el siguiente artículo.

Palabras claves: agroecología, asociatividad, comercialización, cooperación, integración social, sostenibilidad.

\section{ABSTRACT}

The associativity took a leading role, due to times of crisis, in this way it constituted a contribution to the problems of small producers in the cooperative and associative sector of the popular and solidarity economic model in the current market. This article is based on the analysis of strategies to promote the commercialization and marketing of agro-ecological foods that faced the new demands between producers and

\footnotetext{
${ }^{1}$ Magíster en Gerencia de Proyectos Educativos y Sociales. Profesora en la Universidad Tecnológica Indoamérica. Ecuador. Email: lizziepazminio@uti.edu.ec / ORCID: https://orcid.org/0000-0002-5003-1580

2 Máster Universitario en Diseño y Gestión de Proyectos Tecnológicos. Director del Centro de Transferencia de Tecnología e Innovación de la Universidad Tecnológica Indoamérica. Ecuador. Email: jorgealvarez@uti.edu.ec / ORCID: https://orcid.org/0000-0002-3143-7059
} 
consumers. This work was developed with a theoretical character, for which an extensive bibliographic study of publications of the last five years was carried out, referring to associativity strategies. The results revealed the popular and solidarity sector investigated resorted to associativity as a collective model to increase their equitable competitiveness among the autonomous groups of small and medium producers. In addition, They adopted strategies of continuous improvement for cooperative work in a network, training and social integration, technological innovation and the change of agricultural practices. Hence, it is concluded that associativity constituted a strategy of fundamental importance towards the cooperative and comprehensive development of collective groups in the context investigated to be developed in the following article.

Key words: agroecology, associativity, commercialization, cooperation, social integration, sustainability.

\section{INTRODUCCIÓN}

La asociatividad ha tomado un papel protagónico y más aún tras la pandemia COVID19, de tal manera que constituye un aporte a los problemas de las comunidades y familias campesinas que se dedican a las labores agrícolas, quienes garantizan la producción de alimentos agroecológicos para el abastecimientodirecto del campo a la ciudad. Es evidente que tras los cambios económicos, sociales y políticos, a nivel mundial, se ha reflexionado sobre la relación con la naturaleza y la sostenibilidad(Rodríguez E, Holmes y Ramírez G, 2016).

Los nuevos escenarios, así como las exigencias y amenazas competitivas obligan a replantear estrategias de desarrollo organizacional que, desde la asociatividad, se toma como un modelo colectivo que hoy en día se está implementando en diferentes sectores productivos, con el propósito de incrementar y garantizar una competitividad equitativa en las cadenas agro-productivas. Pero existen limitaciones de comercialización y distribución para los productores agroecológicos y familias asociadas, debido a la intermediación de quienes se desenvuelven en un campo competitivo, en desventaja (Zambrano, 2015).

La importancia de mantener un trabajo asociativo, como única forma de impulsar la calidad de la producción rural, depende de la consolidación de los grupos colectivos asociados centrados en la concientización de una alimentación saludable, pero esto ha recibido poca atención en el ámbito rural y urbano debido a que los pequeños emprendedores actúan de manera individual al momento de producir y comercializar los productos; no obstante, al articularse con otros actores que participan y les brindan el apoyo, sean productores, comerciantes e intermediarios, se habla de procesos de asociatividad, como lo menciona (Riveros et al., 2011).

Para Borja et al., (2017), en el Ecuador la asociatividad de las organizaciones forman alianzas entre individuos que se articulan voluntariamente para satisfacer las necesidades desde la colectividad. Las cooperativas son ejemplos de asociatividad, trabajan bajo conceptos, como: compromiso y confianza, lo cual, es importante; pero mantienen respeto por la interdependencia, ya que cada actor tiene autonomía 
gerencial y jurídica (Rodríguez, 2008). La asociatividad surge como una estrategia de cooperación entre pequeñas y medianas organizaciones, con la finalidad de enfrentar las dificultades que trae consigo la globalización, que por un lado brinda oportunidades a nuevos mercados, avances y flujos de información, pero por otro se crean nuevas exigencias competitivas (Liendo, 2013).

Así también como lo dice Martín-Cabello (2013) la globalización se podría comprender como un proceso evolutivo que surge desde las primeras civilizaciones con el apego innato del ser humano al intercambio,aunque tuvo un mayor progreso hasta la actualidad desde la época de la revolución industrial en el siglo XIX.

La globalización es un fenómeno desarrollado en un contexto histórico amplio, por lo que, la competitividad ha sido una de sus consecuencias. Vives (2019), afirma que la competitividad no surge naturalmente, sino, que se cimenta a través de un proceso donde diferentes actores juegan su papel: proveedores, empleados, administraciones locales, regionales y nacionales de los que depende generar competitividad con un servicio o producto que resulte atractivo; con capacidad de venta, rentable y respetuoso con nuestro entorno, cultura e identidad. Tal como lo señalan los autores Massera et al. (2020)sobre los efectos de la globalización y la concentración de las cooperativas agrícolas, acciones y decisiones que deben asumir, en términos de estrategias, estos deben poseer organización interna, relaciones con sus asociados y el fomento de la participación democrática y solidaria para adaptarse al cambio; todo esto, sobre la base de un estudio, exploratorio, realizado en Francia.

Los autores Lara Gómez, Graciela y Pérez Hernández (2020)muestran los beneficios constitucionales que motivan la presencia de las cooperativas y los elementos legales que promueven o restringen su desarrollo y expansión. Mientras que Lopez-Castro (2020)destacalos retos y perspectivas en escenarios de dificultad y retroceso de las actividades económicas, en la región de Pampeana -Argentina durante los últimos años.

Por ello es muy importante que en una cooperativa u organización se establezcan estrategias y canales de distribución, necesarios para cumplir los objetivos, en algunas se habla de cadenas productivas que son "funciones de producción, transformación, comercialización y consumo que realizan diferentes actores, de manera que puedan ser fortalecidas para mejorar su competitividad"(Gottret, 2010, p.17). Sin embargo, el mismo autor menciona que existe una cadena de valor que se contrasta con lo anterior, ya que esta se caracteriza por tener un mayor nivel de confianza, integración y coordinación entre actores para alcanzar una visión común y así desarrollar procesos de innovación y sostenibilidad. La inexperiencia de estas nuevas estrategias y modelos alternativos de organización dentro de la asociatividad para la creación de grupos colectivos permite indagar sobre la necesidad de una aplicación correcta de este mecanismo, por lo que este artículo analiza estrategias para potenciar la comercialización de alimentos agroecológicos que afronten las nuevas exigencias, entre productores y consumidores.

El mundo globalizado hace que nos preguntemos ¿Este es realmente el proceso en el cual nos queremos embarcar para desarrollarnos como una sociedad? si bien, la globalización es una oportunidad para el impulso económico y la conectividad entre 
estados (Fernández,2019) este concepto se cumple solo para quienes se favorecen de estos sistemas que se han ido fortaleciendo masivamente en el mercado y en la economía, siendo como principales el neoliberalismo y el capitalismo manejados en base a conceptos diferentes pero complementarios con el único objetivo de beneficiar a la propiedad privada, volviéndonos de este modo dependientes de un sistema que no es autosostenible sino más bien que promueve la desigualdad, la exclusión y en fin la destrucción del planeta.

Por ello el desarrollo social sostenible reside en compensar las necesidades actuales y futuras de los seres humanos y el medio ambiente, sin pensar únicamente en los factores económicos como suele ocurrir, procurando mantener adecuadamente el medio ambiente (Larco-Pullas, 2019). Por otro lado en la Asamblea General Naciones Unidas (2017) menciona los retos y perspectivas en escenarios de dificultad y retroceso de las actividades las Naciones Unidas establecen que la soberanía alimentaria y agroecológica permiten aumentar los ingresos de pequeños productores, la seguridad alimentaria, el amparo y rescate de los capitales naturales y los medios de sostén en la población rural.

Cabe mencionar que, la soberanía alimentaria junto con la agricultura alternativa como marco general y político en la estructura social de cada país ha cuestionado la insostenibilidad de la actividad agraria industrial; para esto se deben relacionar bien las acciones productivas, por el derecho de los campesinos a producir alimentos sanos, así como la de los consumidores, a poder decidir frente a las verdaderas necesidades de las personas. De la misma forma se debe de salvaguardar los recursos naturales, la biodiversidad y mejorar la resiliencia de los agricultores, que es donde hay una mayor concentración de situaciones de hambre.

También la Organización de las Naciones Unidas para la Alimentación y la Agricultura FAO. (2020) propone algunas ideas concretas para el desarrollo de capacidades de comercio digital e-commerce, de empresas agroalimentarias con el fin de permitir a los agricultores acceder a mercados en línea con los subsecuentes beneficios de recuperación económica, la generación de empleo, el fortalecimiento de cadenas de comercialización y el incremento de la capacidad productiva. Se menciona que en América Latina y el Caribe es la región más cara del mundo para adquirir alimentos sanos y nutritivos, hecho contrastante ya que América Latina tiene la mayor diversidad del mundo en frutas y legumbres (Guerrero,2021).

Además, Gottret (2010) señala que un proceso sostenible es aquel que puede mantenerse durante largo tiempo sin causar agotamiento y daño ambiental de los recursos, destaca también que la sostenibilidad abarca las dimensiones: ambientales, económicas, sociales e institucionales. Aunque muy pocos de estos medios de vida en la sociedad pueden calificarse como sostenibles es importante perseguirlos como una meta para fortalecerlos o mejorarlos.

En México los circuitos cortos de comercialización para la seguridad alimentaria, según Martínez et al. (2021), se basan en la venta directa de productos frescos y de temporada, con mínima intermediación. Se caracterizan por la cercanía geográfica, la participación de unidades de economía familiar, artesanal y reducción de la cadena de valor alimentaria creando más confianza, integración y coordinación para alcanzar 
la visión común y desarrollar procesos de innovación y sostenibilidad. El marco jurídico, económico popular y solidario del Ecuador genera reciprocidad, redistribución, administración doméstica e intercambio a través de la asociatividad, así como del cooperativismo y el emprendimiento, pero las organizaciones adscritas requieren seguimiento y apoyo con la finalidad de mejorar la sostenibilidad y el crecimiento como organización (García y Solarte, 2019).

El Ecuador tiene un alto nivel asociativo, cooperativo y comunitario, señalado en la Ley Orgánica de la SEPS establece a la asociatividad como el mecanismo de cooperación entre organizaciones para afrontar los procesos de la globalización en la economía del país (Asamblea Nacional del Ecuador, 2018) de esta manera se permitirá estrategias inclusivas que se vinculen a las asociaciones y pequeños productores, en mercados dinámicos y colectivos, que ofertan productos agrícolas a través de los circuitos cortos de comercialización con procesos de consumo responsable, precio justo, alimentos sanos y soberanos.

En Ecuador de acuerdo con el Plan Nacional de Desarrollo se plantea el objetivo 6: "Desarrollar las capacidades productivas y del entorno para lograr la soberanía alimentaria y el Buen Vivir rural" (Secretaría Nacional de Planificación y Desarrollo, 2017 , p. 84). Debido a la crisis sanitaria que ha vivido el país, no se ha fomentado dichas capacidades en contextos amigables y en la actualidad hacen falta políticas y lineamientos que permitan realmente este desarrollo, junto con la participación articulada de los actores sociales, las cadenas productivas, los negocios inclusivos, los circuitos alternativos de comercialización y el comercio justo de los ecosistemas.

El Consorcio de Gobiernos Autónomos Provinciales (CONGOPE) organizó un ciclo foro con el tema: "Los retos de una nueva asociatividad urbana y solidaria" donde los panelistas dieron su punto de vista y explicaron como mediante herramientas tecnológicas, enfrentaron la crisis sanitaria, por COVID-19.

\section{Ilustración 1:}

Foro virtual sobre los retos de una nueva asociatividad urbana y rural en tiempos de pandemia

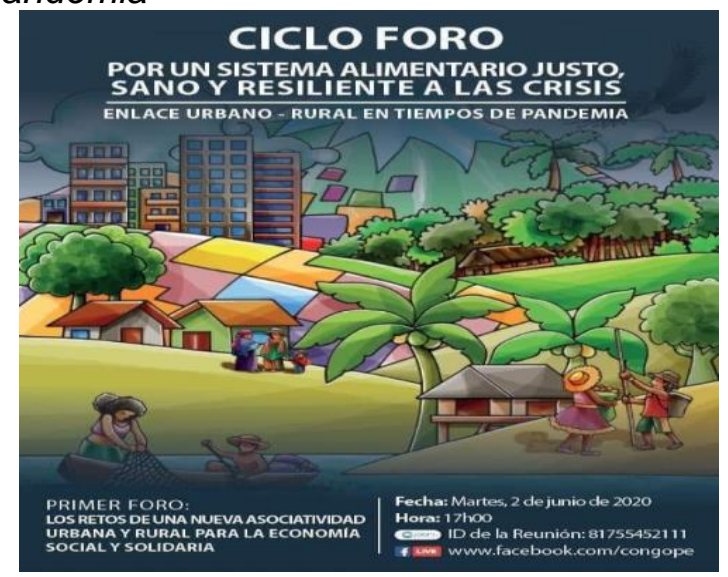

Fuente: CONGOPE, (2020)

Otra institución pública del Ecuador que, durante el 2020, realizó un importante diálogo de opinión acerca de la emergencia sanitaria y las estrategias comerciales de 
las organizaciones del sector popular y solidario fue el Instituto Nacional de Economía Popular y Solidaridad al(EPS), de esta forma brindó información objetiva acerca de la situación de los pequeños productores frente al contexto competitivo, también se dieron a conocer las experiencias y la evolución en el mercado de varios emprendimientos y asociaciones adscritas.

\section{Ilustración 2:}

Webinar: Estrategias comerciales para organizaciones de la EPS Alternativa y desafíos

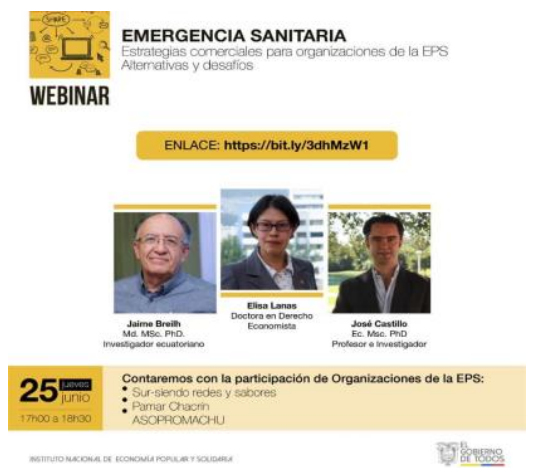

Fuente: EPS, (2020)

En la provincia de Tungurahua Díaz et al. (2017) analiza algunos factores que animan la sostenibilidad de los circuitos de comercialización que a su vez se relaciona con otro estudio de Caicedo, Wendy y Acosta (2020), realizado en la ciudad de Quito, sobre la cadena agro productiva sostenible que gestiona la Cooperativa Sur. Esta Cooperativa ha mantenido el circuito corto de comercialización, desde la actividad agrícola, de esta forma se fomenta el cooperativismo, el desarrollo comunitario y el socio productividad.

Según los autores la socio productividad hace referencia a pequeñas extensiones de terreno, pero con variedad de rotación en el cultivo, lo que permite que la tierra se recupere, esta es considerada como un modelo colectivo basado en los principios de un trabajo liberador, solidario, de control social, autogestión y la conformación de nuevas relaciones sociales, reduciendo así la presencia de intermediarios en beneficio de cinco organizaciones, de productores de familias campesinas, que buscan potenciar las prácticas de una economía social.

Actualmente, en medio de las adversidades de un sistema agroalimentario modernizante, varios actores sociales del colectivo agroecológico de la provincia de Pichincha trabajan, de forma articulada, en espacios de diálogo y acción colectiva para una agenda agroecológica común mediante vínculos de corresponsabilidad, reciprocidad e interdependencia para la zona urbana y rural del país. 
Ilustración 3:

Ecosistema de actores rumbo al Encuentro Nacional del Colectivo Agroecológico del Ecuador

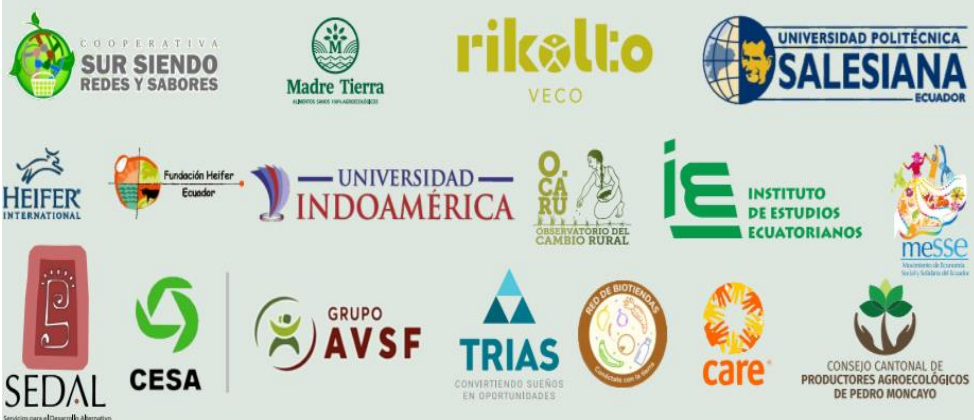

Fuente: Instituciones públicas y privadas, ONG's, investigación y academia del Ecuador

Elaborado por: Cooperativa Sur Siendo Redes y Sabores (2021)

Ciertamente en el Ecuador la profundización de la crisis, por los efectos de la pandemia sobre los patrones de consumo y riesgo, fueron diversos afectando a los grupos más vulnerables y caracterizado por los siguientes aspectos: el cierre de los mercados locales, las compras de pánico frente a la incertidumbre, el enfrentamiento de estrategias de mercadotecnia publicitadas por el internet y la televisión, el sobreabastecimiento de alimentos procesados y ultra procesados, las dificultades de tiempo al momento de elegir y consumir los productos sin verificar la etiqueta dentro de los supermercados (Martin-Neuninger y Ruby, 2020).

Cabe mencionar que el Ecuador ha enfrentado situaciones difíciles por las malas decisiones políticas del último gobierno y la emergencia sanitaria que actualmente ha incrementado la pobreza en el país, lo que ha provocado un decrecimiento en la economía; es así como, las diferentes organizaciones agrícolas se han visto obligadas a desenvolverse en un campo competitivo. Por ello es fundamental que los procesos asociativos y cooperativos permitan el desarrollo armónico de las cadenas de valor, mediante una relación directa con el consumidor final y que estas puedan ser fortalecidas con el acompañamiento de los actores sociales participantes, para mejorar la competitividad.

Las estrategias asociativas se han ido ajustando a los nuevos escenarios gracias a los distintos enfoques de comercialización y ampliación que manejan, cabe destacar que muchas veces sin recursos, sin infraestructura, esto ha conllevado a un trabajo voluntario para no solo conseguir ingresos, sino para que se siga un proceso que cree condiciones de sostenibilidad para generar un servicio de calidad al consumidor.

\section{METODOLOGÍA}

El presente estudio es de carácter teórico de alcance exploratorio-descriptivo, para el cual se realizó una amplia revisión bibliográfica de artículos, que analizó las características y estrategias asociativas de los últimos cinco años y como estas contribuyen $\mathrm{o}$ afectan a las organizaciones sociales de incidencia, dentro de su contexto de desarrollo. Para conseguir el objetivo planteado se realizó, 
principalmente, una búsqueda booleana (AND, OR) de artículos identificados con las palabras claves como "estrategias asociativas", "comercialización productos agroecológicos", "sostenibilidad", en las bases Researchgate, Scopus Scielo y Google Académico, entre otras, de repositorios de instituciones públicas y privadas.

La búsqueda se realizó desde marzo a octubre del 2021, se sistematizó el aporte científico de 15 artículos, mediante un análisis documental y comparativo utilizando criterios de inclusión y exclusión de las categorías: fuente, país, sector, variables, muestra, estrategia y producto, de las cuales las tres últimas permitieron ampliar a detalle la revisión completa de los textos obteniendo inicialmente 43 características vinculadas a la asociatividad y agrupadas en orden de mayor frecuencia, luego se agruparon en 10 estrategias y finalmente 5 cinco estrategias fundamentales en las que se basa la asociatividad, información que sirvió de ayuda para la discusión.

\section{RESULTADOS}

Con relación a la crisis sanitaria, de las últimas décadas, se vuelve imprescindible mejorar la asociatividad, es por ello por lo que la selección y comparación de documentos, mediante criterios de inclusión y exclusión, permitieron establecer las principales características y estrategias asociativas que facilitaron una implantación en las organizaciones, tanto en la economía, en lo popular, en lo social y solidaria de las investigaciones citadas, ver la TablaN ${ }^{\circ} 1$.

Dentro de las prácticas de integración social se establecen las formas de asociatividad, entre ellas: presta mano, mingas, alianzas, lazos sociales y redes de servicios de grupos asociados por afinidad para el desarrollo socio-productivo, mediante elementos críticos de éxito como: participación, compromiso, transparencia, persistencia y liderazgo que acompañan los procesos asociativos por afinidad ideológica, autogestión social y cooperativa. Las alianzas y la articulación del sector rural y urbano son fundamentales para generar prácticas de confianza, ampliar las posibilidades de crecimiento y una competencia equitativa. La agricultura alternativa, cultural y sostenible es otra característica que consideran factores endógenos y exógeno para comunicar, promover y difundir la asociatividad productiva, natural, turística y de valores. De ahí que, mediante políticas de fomento que aporten a la sostenibilidad, el crecimiento productivo y la comercialización de base agroecológica familiar, se ha logrado la implementación de estrategias de marketing digital.

Es importante destacar la formación y capacitación técnica en ambientes que fomentan las relaciones sociales, la transferencia de conocimiento asociado al capital humano, social, natural y físico, sistemas de producción integrados; es decir la forma como los campesinos se organizan en sus cultivos y crianzas, junto con las buenas prácticas agrícolas como base para la sostenibilidad. Esto permitirá la autogestión social, económica y cooperativa como hace referencia (Martínez Salvador et al., 2021) el caso M100 ha fortalecido procesos de gestión organizacional con resultados significativos para el rescate del abasto y la seguridad alimentaria, con autonomía. 
Tabla 1:

Estrategias Asociativas

\begin{tabular}{|c|c|c|c|c|c|}
\hline \multirow[b]{2}{*}{ Producto / Autor } & \multicolumn{5}{|c|}{ Estrategiasde Asociatividad } \\
\hline & 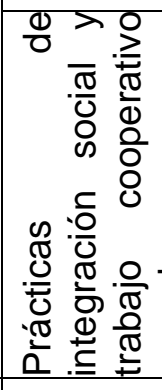 & 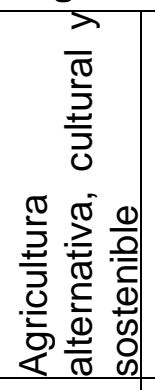 & 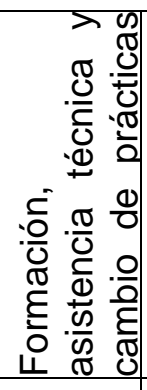 & 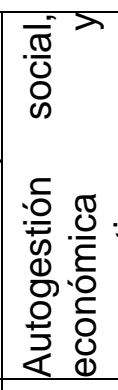 & 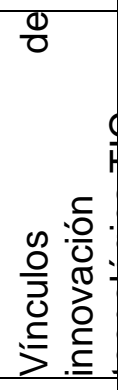 \\
\hline $\begin{array}{l}\text { Esquemas de mercado asociativos sostenibles y } \\
\text { competitivos. (Parrales et al., 2021) }\end{array}$ & - & - & & - & \\
\hline $\begin{array}{l}\text { Modelo de abasto y seguridad alimentarios caso } \\
\text { M100. (Martínez Salvador et al., 2021) }\end{array}$ & & - & 口 & & \\
\hline $\begin{array}{l}\text { Compromiso, transparencia, persistencia y } \\
\text { liderazgo.(Caicedo, Wendy y Acosta, 2020) }\end{array}$ & - & प & प & 口 & \\
\hline $\begin{array}{l}\text { Cadena agropecuaria y turismo asociativo y } \\
\text { cooperativo.(Nieto \& Uniagustiniana, 2020) }\end{array}$ & - & प & प & U & \\
\hline $\begin{array}{l}\text { Conocimiento, } \quad \text { relaciones sociales y } \\
\text { productividad.(Sala, ALO y Nieves, 2019) }\end{array}$ & - & प & प & प & \\
\hline $\begin{array}{l}\text { Gestión asociativa capital humano, social, natural, } \\
\text { físico. (Bobadilla Díaz et al., 2019) }\end{array}$ & - & प & & 口 & \\
\hline $\begin{array}{l}\text { Conocimiento asociado al capital natural } \\
\text { sostenible BPA. (Fonseca-Carreño et al., 2019) }\end{array}$ & - & प & प & & \\
\hline $\begin{array}{l}\text { Producción de base agroecológica familiar. (De } \\
\text { Almeida et al., 2019) }\end{array}$ & - & 口 & प & & \\
\hline $\begin{array}{l}\text { Afinidad ideológica, autogestión social y } \\
\text { cooperativa. (Espelt et al., 2018) }\end{array}$ & - & & & U & प \\
\hline $\begin{array}{l}\text { Agricultura alternativa y el agronegocio urbano y } \\
\text { rural. (Palmisano, 2018) }\end{array}$ & - & 口 & प & & \\
\hline $\begin{array}{l}\text { Expansión agroecológica y soberanía alimentaria. } \\
\text { (Gortaire, 2017) }\end{array}$ & - & 口 & प & & \\
\hline $\begin{array}{l}\text { Formas de asociatividad, alianzas sociales y redes } \\
\text { de servicio. (Borja et al., 2017) }\end{array}$ & - & 口 & & & \\
\hline $\begin{array}{l}\text { BPA y comportamiento del consumidor (Díaz et al., } \\
\text { 2017) }\end{array}$ & - & " & घ & & \\
\hline $\begin{array}{l}\text { Redes, clústers y grupos asociados por afinidad. } \\
\text { (Fajardo Vaca et al., 2016) }\end{array}$ & - & " & & 口 & \\
\hline $\begin{array}{l}\text { Factores endógenos y exógenos que promueve la } \\
\text { asociatividad. } \\
\text { (Naclerio, Alejandro y Trucco, 2015) }\end{array}$ & $\square$ & - & & & 口 \\
\hline
\end{tabular}

Fuente: Investigación bibliográfica

Elaborado por: Los Autores

Dichas estrategias son una contribución a los problemas que presentan estas organizaciones en el mercado actual, al mantener el trabajo asociativo como única 
forma de impulsar la calidad, la competitividad y la producción agroecológica que generen mejores condiciones de subsistencia, entre el campo y la ciudad.

\section{DISCUSIÓN}

El mercado actual de productos agroecológicos ha experimentado cambios acelerados luego de la pandemia, ahora es mayor la demanda de este tipo de agricultura, pero los procesos de certificación de base agroecológica se hacen imprescindibles para ser competitivos y con mayor acceso a mercados diferenciados de los convencionales que brinden seguridad al consumidor y comercialicen alimentos sanos sin agrotóxicos.

En la investigación se identificaron estrategias de carácter asociativo, cooperativo, integrador, tecnológico, de formación y que se enmarcan en las buenas prácticas que mejoran la gestión productiva y comercial de los productos agroecológicos y de las organizaciones locales y nacionales de grupos colectivos de la economía social y solidaria.

Los estudios han adoptado estrategias de mejora continua para el trabajo cooperativo en redes, clústers y grupos asociados, por afinidad, como pilotos 0 modelos de producción colectiva quienes ha mediado nuevas propuestas y relaciones sociales, en base a los principios de una economía liberadora, solidaria, de control social y de autogestión.

Dentro de las prácticas de integración social, de los emprendimientos asociativos, se destaca que en el Ecuador existen varios proyectos colectivos que han logrado, mediante un trabajo voluntario y cooperativo, conectar con personas y actores que tienen intereses y objetivos comunes como: la asociatividad, el cooperativismo, la agroecología, el cuidado de la tierra; así también, la seguridad y soberanía alimentaria, el consumo responsable, el desarrollo sostenible, el medio ambiente, entre otros.

Se promueven las relaciones entre productores y consumidores mediante acciones de liderazgo, organización, toma de decisiones, resolución de conflictos, trabajo cooperativo con transparencia y participación democrática del colectivo, para enfrentar los desafíos del sistema político, globalizado y económico. Todo lo anterior debido a la primacía de la industria agroalimentaria, la expansión de los supermercados, la proliferación del comercio informal y migrante que ha deteriorado los ingresos dignos de las organizaciones y familias rurales.

Se destacan estudios relacionados con la promoción mediante actividades sociales y culturales, asistencia técnica y la capacitación, para que se haga dinámico el proceso asociativo entre productores y consumidores, integrando a los más jóvenes para que se sientan parte de este cambio y aporten en la construcción y mejora de los ecosistemas sostenibles.

Finalmente, se concluyó que la asociatividad se basa en algunas estrategias fundamentales en las que se han encontrado las acciones integración social y cultural, el trabajo cooperativo en red, la capacitación y asistencia técnica de prácticas alternativas y sostenibles, la autogestión cooperativa y la innovación tecnológica, para 
enfrentar las barreras y limitaciones de la intermediación en los procesos de comercialización, asumiendo nuevos retos y roles de manera conjunta para el trabajo asociado, integral, cooperativo y voluntario de las organizaciones y movimientos sociales locales y nacionales de la economía solidaria, generando mejores condiciones de vida y de subsistencia de productores y consumidores en el campo y la ciudad.

\section{REFERENCIAS BIBLIOGRÁFICAS}

Asamblea General Naciones Unidas. (2017). Informe de la Relatora Especial sobre el derecho a la alimentación. 01059 http://www.greenpeace.org/argentina/Global/argentina/2017/3/ONU_agro_G1701790.pdf

Asamblea Nacional del Ecuador. (2018). Ley Orgánica de la Economía Popular y Solidaria y del Sector Financiero Popular y Solidario. In Registro Oficial 444de 10 -mayo-2011: Vol. Reformado (Issue 7).

Bobadilla Díaz, P., Puente de la Vega, M. P., \& Fernández Escobar, R. (2019). La influencia de la asociatividad en el desarrollo de oportunidades productivas: el caso de cuatro asociaciones agropecuarias de la región Moquegua - Perú. Debates En Sociología, 48, 65-102. https://doi.org/10.18800/debatesensociologia.201901.003

Borja, C., Alarcón, G., \& Quizhpe, V. (2017). La asociatividad de las organizaciones sociales del sector subtrópico de la provincia Bolívar. Observatorio de La Economía Latinoamericana, 233, 1-16.

Caicedo, Wendy y Acosta, B. (2020). Factores críticos de éxito de las asociaciones dentro de la Economía Popular y Solidaria [Universidad Técnica del Norte]. http://repositorio.utn.edu.ec/jspui/bitstream/123456789/10767/2/02 ICO 752 TRABAJO GRADO.pdf

De Almeida, E. W., Stumpf, M. T., Porto, C. R. P., Rolon, A. S., \& Trentin, G. (2019). Potencial agroecológico de propriedades agrícolas familiares do município de São Lourenço do Sul, Rio Grande do Sul. Revista Verde de Agroecologia e Desenvolvimento Sustentável, 14(4), 546553. https://doi.org/10.18378/rvads.v14i4.6551

Díaz, J. C., Chauca, M. P., \& Ceballos, S. T. (2017). Agroecological short circuits of marketing in Ecuador. Idesia (Arica), 35(3), 71-80. https://doi.org/10.4067/S0718-34292017005000302

Espelt, R., Peña-López, I., \& Rodríguez, E. (2018). Alternative economy or technopolitics? Activism in food consumers' cooperatives. CIRIEC-Espana Revista de Economia Publica, Social y Cooperativa, 93, 293-318. https://doi.org/10.7203/CIRIEC-E.93.9460

Fajardo Vaca, L., Fernández Ronquillo, M., Vásquez Fajardo, C., Toscano Ruiz, D., \& Fajardo Vaca, L. (2016). La Asociatividad Microempresarial, como mecanismo productivo sostenible: Caso Cantón Milagro - Ecuador TT - The microenterprise association, as a sustainable production mechanism: Canton Milagro case - Ecuador. Universidad, Ciencia y Tecnología, 20(78), 4-13.

FAO. (2020). Sistemas alimentarios y COVID-19 en América Latina y el Caribe: Reconstrucción con transformación: un balance de medio término. In Boletin $N^{\circ} 17$ (CEPAL, p. 21).

Fernández, H. (2019, 24 de julio). Qué es la globalización. Economía TIC.https://economiatic.com/globalizacion/\#

Fonseca-Carreño, N. E., Salamanca-Merchan, J. D., \& Vega-Baquero, Z. Y. (2019). La agricultura familiar agroecológica, una estrategia de desarrollo rural incluyente. Una revisión. Temas Agrarios, 24(2), 96-107. https://doi.org/10.21897/rta.v24i2.1356

García Ibarra, V. J., y Solarte Solarte, M. L. (2019). La asociatividad y el emprendimiento. Visión Empresarial, 9, 25. https://doi.org/10.32645/13906852.867

Gortaire, R. (2017). Agroecología en el Ecuador. Proceso histórico, logros, y desafíos. Antropología Cuadernos de Investigación, 12, 12-38. https://www.redalyc.org/pdf/837/83710302.pdf

Gottret, M. (2010). Orientación estratégica con enfoque de cadena de valor para la gestión de empresas asociativas rurales. In CATIE (Ed.), Centro Agronómico Tropical de Investigación y Enseñanza (CATIE) (Vol. 4, Issue 3, pp. 57-71). http://marefateadyan.nashriyat.ir/node/150

Guerrero, R. (2021, 12 de octubre). Desafíos Post COVID-19 personas, tecnología y el camino hacia la sostenibilidad y resiliencia de la OEPS [ponencia]. Inclusión Financiera Digital, Transparencia y Gobernanza, Quito, Ecuador. https://jornadas.seps.gob.ec 
Lara Gómez, Graciela y Pérez Hernández, C. C. (2020). Retos y perspectivas para el cooperativismo mexicano. Deusto Estudios Cooperativos, 16, 163-182. https://doi.org/10.18543/dec-162020pp163-182

Liendo, M. M. A. (2013). Asociatividad. Una Alternativa para el desarrollo y crecimiento de las Pymes. In E. de E. Instituto de Investigaciones Económicas (Ed.), Encyclopedia of Earth Sciences Series (p. 1096). 2001. https://doi.org/10.1007/978-1-4020-4399-4_33

López-Castro, N. (2020). Estrategias del cooperativismo agrario frente a las transformaciones sociales y productivas recientes. Cooperativismo \& Desarrollo, 28(117), 1-32. https://doi.org/10.16925/2382-4220.2020.02.03

Martín-Cabello, A. (2013). Sobre los orígenes del proceso de globalización. Sobre Los Orígenes Del Proceso de Globalización, 1(1), 7-20. https://doi.org/10.17502/m.rcs.v1i1.22

Martin-Neuninger, R., \& Ruby, M. B. (2020). What Does Food Retail Research Tell Us About the Implications of Coronavirus (COVID-19) for Grocery Purchasing Habits? Frontiers in Psychology, 11(June), 1-4. https://doi.org/10.3389/fpsyg.2020.0144.

Martínez Salvador, L. E., Hernández, L. G., \& Alvarado Ramírez, D. (2021). Cadenas Cortas de Comercialización y seguridad alimentaria: el caso de El Mercado el 100. Problemas Del Desarrollo. Revista Latinoamericana de Economía, 52(206), 197-220. https://doi.org/10.22201/iiec.20078951e.2021.206.69732

Massera, M. L., Cuatrín Sperati, E., \& Locher, M. V. (2020). El cooperativismo agrícola en el contexto de globalización. Un estudio exploratorio en el sur de Francia. Eutopía. Revista de Desarrollo Económico Territorial, 18, 95-116. https://doi.org/10.17141/eutopia.18.2020.4666

Naclerio, Alejandro y Trucco, P. (2015). Construir el desarrollo con Políticas Públicas: Asociatividad, Tecnología e Innovación Productiva. El Caso del Programa Sistemas Productivos Locales. Documentos y Aportes En Administración Pública y Gestión Estatal, 24, 33-65. https://doi.org/10.14409/da.v0i24.4809

Nieto, A., \& Uniagustiniana, U. (2020). Productividad agrícola y turismo: ejes de articulación para el cooperativismo con jóvenes campesinos Agricultural productivity and tourism: articulation shafts for the cooperativism with youth peasants. April.

Palmisano, T. (2018). Las agriculturas alternativas en el contexto del agronegocio. Experiencias en la provincia de Buenos Aires, Argentina. Reis, 82, 291. https://doi.org/10.2307/40184061.

Parrales, L., Basurto, C., Cruz, M. I., \& Ponce, J. A. (2021). Asociatividad, cadena de valor e impacto de ambas. 8, 392-413.

Rodríguez E, Holmes y Ramírez G, C. J. (2016). Sustainability analysis of the strengthening processes of a rural association: Asomora a case study. Revista de Ciencias Agrícolas, 33(1), 09-21.

Rodríguez, F. B. (2008). Las redes empresariales y la dinámica de la empresa. Innovar.

Sala, ALO y Nieves, V. (2019). Estrategia asociativa y productiva para el sector piscícola en Cumbal y Potosí, Nariño. Revista Veterinaria y Zootecnia (En Línea), 33(7), 12-13. https://doi.org/10.17151/vetzo.2019.13.2.4.

Secretaría Nacional de Planificación y Desarrollo. (2017). Plan Nacional de Desarrollo 2017-2021-Toda una Vida. http://www.planificacion.gob.ec/wp-content/uploads/downloads/2017/10/PNBV-26OCT-FINAL_OK.compressed1.pdf

Vives, X. (2019). Competitividad en las empresas y los destinos del turismo. In Artículo Tourism Business \&Planning (pp. 1-3). www.c4tourism.com

Zambrano, G. M. H. (2015). Estrategias de Asociatividad para el sector Cafetalero de la Federación Regional de Asociaciones de Pequeños Cafetaleros Ecológicos del Sur de la Cuidad de Loja. Escuela Politécnica Nacional de Ecuador. 